\title{
Robots Moving out of the Laboratory - Detecting Interaction Levels and Human Contact in Noisy School Environments
}

\author{
Tamie Salter, Kerstin Dautenhahn \& René te Boekhorst \\ Adaptive Systems Research Group, School of Computer Science, \\ University of Hertfordshire, College Lane, \\ Hatfield AL10 9AB, United Kingdom \\ E-mail t.salter|k.dautenhahn|r.teboekhorst@herts.ac.uk
}

\begin{abstract}
To achieve natural human-robot interaction robots will need to distinguish humans from other parts of the environment. In this paper we investigate how infrared sensors currently being used on a mobile robot can be used to distinguish human interaction. Different from previous work, that had been conducted under laboratory conditions involving selected children, the current study took place in noisy school environments with a mix of children. Also, while in previous work each child was only exposed once to the robot, in the current longitudinal study each child encounters the robot five times. The technique that we developed previously for detecting human contact still proved to be reliable, however results are not as clear-cut, due to noisy and rather unstructured environments that interfered with the robot's sensor readings. We discuss expected as well as unexpected results in light of the challenge to develop robots that can operate under real-life conditions.
\end{abstract}

\section{Introduction}

The work presented here is part of the AuRoRA project [1]. The AuRoRA project investigates how robots can be used in a therapeutic and educational context with autistic children. Robots are increasingly being used in therapy, assistive technology and rehabilitation e.g. $[3,2,9,4]$. Methods that will enable easy communication between robots and humans are essential in all these areas. Typically communication or interaction in robot-human scenarios is achieved through vision (cameras), sound (audio) or touch (push down buttons) $[13,17,14]$. Some researchers are now looking more closely at touch as an important form of communication between robots and humans especially those involved with children and therapy $[12,18,3]$. Work carried out within the AuRoRA project by Salter et al. has been investigating a novel way for robots to detect and distinguish human interaction (in our case with children) $[12,6,5]$. A technique has been introduced whereby infrared sensors located on a mobile robot are used to measure distances in the environment and record close contact or interactions from humans. The robot detects static objects in the environment and avoids them. Thus, it does not come into close contact with static obstacles and does not record them as interactions or contact. Any infrared reading less than $2 \mathrm{~cm}$ is recorded as interaction or contact from a human. This enables natural touch from the child to be recorded without any need for them to push down and exert force in a specific area as is the case with push down buttons.

\section{Investigating different pat- terns of behaviour}

To design robots that can adapt, we first need to know what features of human behaviour the robot should adapt to. To approach this we have started by investigating how different types of typically developing children play with the same robot so that we can learn about patterns of interaction from these children and then compare them to that of the very complex behaviour of autistic children. Salter et al. have shown that patterns of interaction from different children's behaviour can be detected by the robot's sensors $[6,12]$. Their preliminary work was carried out under laboratory conditions with preselected children that exhibited one of three very distinct personality types, the personality type was later shown to be reflected in their behaviour towards the robot. The personality types were rated jointly by the children's parents and the experimenter. Children of type A exhib- 


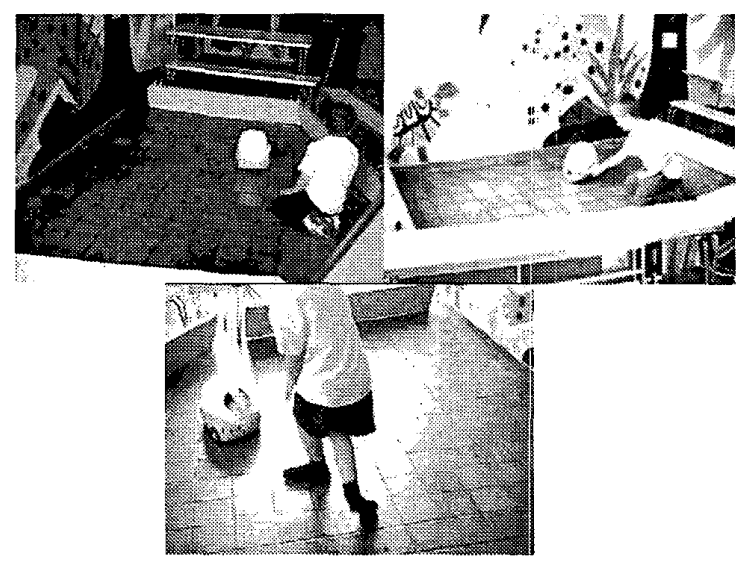

Figure 1: Diagram showing different children playing with the robot with eyes present and without in the 'pen'. Note the different lighting conditions.

ited very boisterous, rough behaviour, type B children showed 'typical', average behaviour, and type C exhibited very shy or cautious behaviour. The longitudinal study introduced here took this method and applied it in more natural conditions with children that were not preselected but just children from an ordinary class at a typical school. As with the previous work $[12,6,5]$ the aim was to see if the sensor readings from the mobile robot used could distinguish human interaction. If so, could these interactions later be analysed so as to distinguish the personality type of a child (A, B or C) that was interacting with the robot? Note, that these children were not preselected and so the task would be harder to achieve.

\section{Experimental setup}

A commercially available medium sized robot was used (see Fig. 1) [16]. The robot came equipped with a ring of 15 infrared sensors located around its rim. Measurements from these sensors are used to record the children's interactions. Autism is four to five times more likely to occur in a boy than a girl. All the subjects were male (we did not plan to address gender differences) and 5-8 years old. The study included 7 typically developing children and one child diagnosed with autism. The children were not preselected, the experimenter rated the category the child appeared to fit: either type A; boisterous, rough, naughty, type $\mathrm{B}$; average, usually well behaved or type C; shy, quiet and cautious. This was based on the experimenter's point of view and came from general observations of the child's behaviour such as how confident the child appeared, how much they questioned the experimenter etc. $^{1}$ Different from the previous study in the lab $[6,12]$ the processing power on the robot had been greatly improved. This enabled sensor data to be written more often and also enabled the network lead to be removed $^{2}$ leaving the robot completely autonomous. The study was carried out in the school's dining room on a tiled floor. A pen was made by placing benches on the floor next to each other (see Fig. 1). The size of the pen was approximately $3 \mathrm{~m}$ by $2 \mathrm{~m}$. In total five experiments were carried out for each child. At the beginning of each trial the children were instructed to enter the pen and do whatever they liked. The robot carried out the same basic obstacle avoidance behaviour regardless of the children's activities. The first three experiments were identical to each other; these experiments lasted for 5 minutes and the robot performed slow obstacle avoidance. Experiment four was identical to experiments one to three except eyes were added to the robot (plastic stickers placed at the front of the robot, see Fig. 1). Experiment five was the same as number four in that the eyes were left on but the robot's behaviour was slighted modified: the speed of the obstacle avoidance was increased. Experiment five lasted for 4 minutes (a shorter length was suggested by the teacher). To cope with the increase in speed the size of the pen was enlarged (to about $4 \mathrm{~m}$ by $2.5 \mathrm{~m}$ ) to enable the robot to travel at a high speed without constantly stopping and turning as it had reached the side of the pen.

Note the original plan was to conduct a longitudinal study with five identical sessions to see the effect of repeated exposure on children's interactions with the robot. However after three trials all the children had clearly lost interest in the robot; they either did not interact with the robot at all, or diverted their interest away from the robot (such as speaking to the experimenter) even if still touching the robot. ¿From an ethical standpoint we did not want to bore the children, this was reinforced by the teacher's opinion. We therefore introduced two different experimental conditions in a fixed order, since we did not intend to make comparisons among them. Thus, the main purpose of conditions 4 \& 5 was to regain the children's interest.

\footnotetext{
${ }^{1}$ In future studies the classification will be confirmed by an independent evaluator.

2 The network lead had been required in the previous trial to link the robot to another computer via a network for extra processing power which enabled the robot carry out both obstacle avoidance and write data to a file.
} 


\section{Data \& Data Analysis}

The data on interactions was obtained from the robot's sensor readings. These sensor readings are written to a text file onboard the robot. Touches or interactions (i.e. close proximity less than $2 \mathrm{~cm}$ distance) were recorded 16 times per second. These records characterise the interaction pattern of that particular child with the robot for that particular session. Three different techniques to analyse the robot's sensor data have been considered. (1). Clustering the different children's profiles (overall interaction levels from the 15 infrared sensors). Cluster analysis [19] was performed on a matrix containing these profiles. This shows which children were similar in terms of sensor activation patterns. This analysis was only applied to the set of the first four sessions. This was because they lasted the same length of time and the same behaviour was exhibited by the robot. (2). Calculating the interaction levels from overall sensor data. We investigated if there were any significant differences in the levels of sensor activation e.g. did active children produce higher activity levels under certain conditions? (3). Time series data from the sessions. Graphs were produced that show the dynamics of the sessions. Patterns of activity can be seen from these graphs.

\section{Results}

All of the children had their own unique way of playing with the robot. All of the children except one (Tom) appeared to enjoy the experience and actively engaged with the robot in their own way. Tom simply sat and watched with robot. Thus, his data was not included in any of the statistical analysis or discussion. Behaviour with the robot ranged from cautious and wary to confident and physically aggressive. All of these findings follow that of the previous work $[6,12]$. A noted difference from previous work was that at times the robot got 'stuck' in the corner during the trials, this never happened during previous trials in the lab.

As was previously found, sensor data clearly highlights the differences in the way the children played with the robot. Following are details of the results from the statistical analysis performed on the sensor data. (1). Cluster analysis of the sensor data showed encouraging results. Despite misclassification from the robot getting stuck, see section 'Unexpected Results', there still appear to be patterns in the way different types of children play with the robot. Even though the children had not been preselected to show clear



Figure 2: Diagram showing clustering results from 8 children's sessions 1 to 4 , the personality category A, $\mathrm{B}$ or $\mathrm{C}$ is indicated next to name. Note data from session 'Joe 1' is missing due to robot malfunction.

'personality types', making the task of categorising them harder, there do appear to be clusters of children. Clusters of children on the diagram indicate children who activated the sensors in a similar way. These clusters seem to match that of the psychological grouping, or personality types, they had been placed in by the experimenter (see Fig. 2). This indicates that children can be categorised into personality types on the basis of sensor activations as was found in the previous work $[6,12]$. (2). Overall interaction levels - see next subsection 'unexpected results'. (3). Time series of the sessions were produced that show the different patterns of play over time. These diagrams show the dynamics of the sessions and a great difference between the most active children and passive children (see Fig. 3 for examples). We are currently working on statistical techniques to utilise the patterns shown in these time series diagrams. These diagrams have been valuable in helping analyse unexpected high interaction levels, see next section.

\section{$5.1 \quad$ Unexpected Results}

The overall interaction levels did not show expected results, which also clearly demonstrated the value of longitudinal studies with repeated exposure of children to robots. With all of the children except one (Sam) it was the experimenter's strong impression 




Figure 3: Diagram showing time series for each of the 5 sessions of the most active child (Walter) and the most passive child included in analysis (Sam). The horizontal axis reflects time and the vertical axis shows activity on the 15 IR sensors.

that the children became successively bored of the robot over the first 3 sessions (Sam seemed very cautious in the first 3 sessions and then seemed to grow in confidence with the robot, see Fig. 3 for his time series results). This was also confirmed by checking video footage of the sessions. It was visible that the children stopped interacting with the robot as much in later sessions and did not appear to pay as much attention to the robot over time. Adding the eyes to the robot in session 4 did give an initial novelty factor but this soon wore off and the children appeared bored again. Speeding the robot up in session 5 did appear to raise the interest of the children and they did appear to interact with the robot more. It was therefore expected to see a general decrease in interaction levels measured by the sensor readings in sessions 1 to 3 , then perhaps a slight increase in levels in session 4 and finally a noticeable increase in interaction levels in session 5. Results of the overall interaction levels from the sensor readings did not show this (see Fig. 4). They showed erratic readings that did not appear to match what had happened in the sessions.

To investigate this further, timed sensor readings were matched to timed video data. It was found that the robot getting 'stuck' in the corner of the pen was affecting the overall interaction levels. Getting 'stuck' can be characterised by the robot moving into

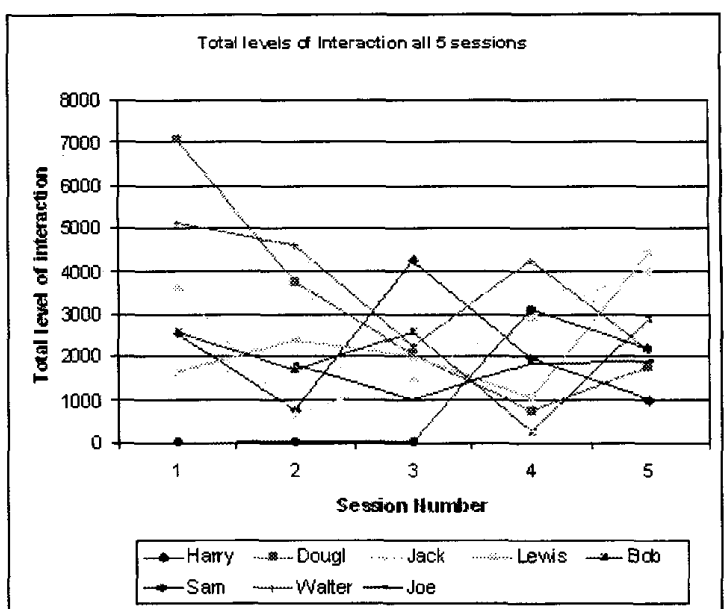

Figure 4: Diagram showing overall interaction level for each child at each session. Note data from 'Joe 1' is missing due to robot malfunction.

and hitting a side of the pen and, despite the wheels turning, the robot not being able to free itself. One clear example of unexpected interaction levels can be seen in the case of Harry, see Fig. 5.

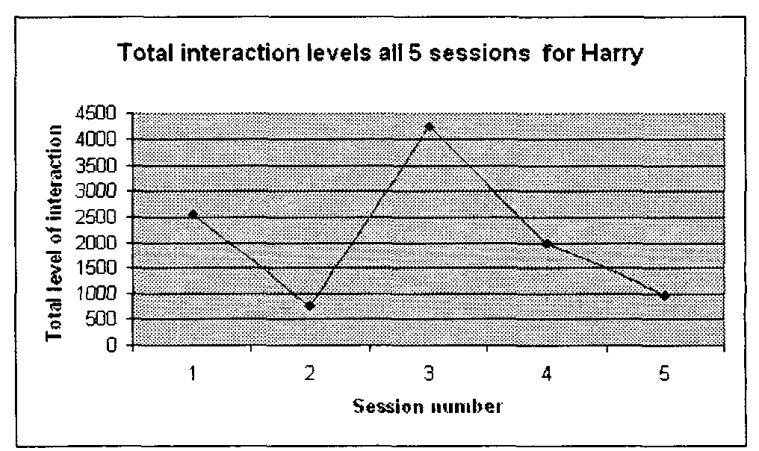

Figure 5: Overall interaction levels for Harry. Unexpected high interaction level can be seen in session 3.

Harry had become bored of the robot over the first 3 sessions, yet the interaction level for his third session was much higher than that of the previous two sessions. After reviewing video data it was discovered that the robot had got stuck three times in Harry's third session causing misclassification in the robot's data. The times the robot became stuck can clearly be seen from the time series graphs, see Fig. 6 . 


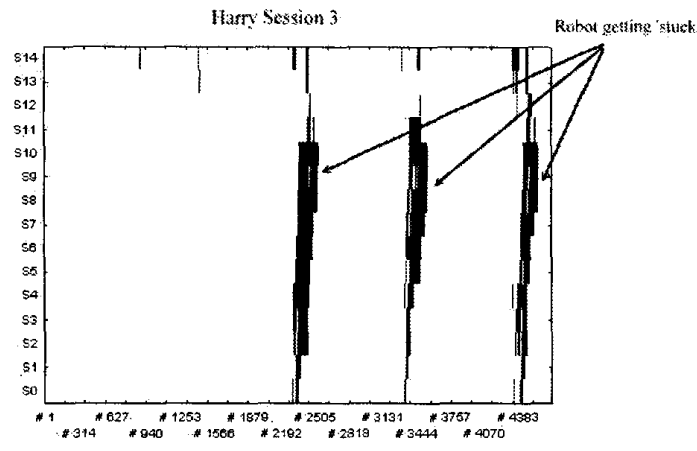

Figure 6: Time series showing session number 3 for Harry.

New experiments have been conducted that study in more detail how the physical environment can affect the robot's sensor readings [7]. Reasons for the robot getting stuck may possibly include lighting conditions affecting sensor readings (see Fig. 1 for examples of different lighting conditions). Also, the angles created by the walls of the 'pen' where the experiment was conducted may have caused problems. It has been found that angles less than 90 degrees possibly confuse the robot about which way it can turn, see [7] for further details.

\section{Discussion}

We previously introduced a technique to detect and record human contact and to make classifications of the 'personality type' from the sensor information. However, using this technique in a more natural environment made this harder than in previous studies. This was due to the robot getting 'stuck' and misclassification's being made from sensor data. Reasons for the robot getting stuck might include natural lighting conditions affecting sensor readings and also the shape of pen in which the experiment was conducted, see [7] for a more detailed analysis of the environment affecting sensor readings. It appears that despite misclassification of sensor readings when the robot got stuck, there are still patterns in the way different types of children play with the robot. As such, clustering results of the children's data did not seem unduly affected, however there was a considerable effect on the overall interaction levels resulting from misclassifications.

Another finding was that the children get bored of the robot over time if it is exhibiting the same be- haviour. This can be seen from video footage and matched the impression of the experimenter. This confirms findings by Kanda et al. [15] that the novelty effect wears out over time. The effect from the eyes being placed on the robot (experiment 4) was only minor. The children seemed to discuss the robot in a more animal like way with the eyes on (e.g. the first child in this experiment asked the experimenter "Is it a cat or a dog?"), but their behaviour toward the robot did not seem to change dramatically and there did not seem to be any noticeable change in the sensor data. Speeding the robot up (experiment 5) did seem to have an effect on the children. Their behaviour whilst playing with the robot changed, they raced the robot which was behaviour only seen in this experiment. They seemed more interested in the robot again and generally appeared to be more engaged and active. This appears in time series data, there seems to be a greater scattering of interactions over the final session's diagram for the group as a whole.

We would therefore conclude that there is real evidence for using infrared sensors to enable or enhance robot-human interaction interfaces even in natural noisy environments. Infrared sensor data does seem to give a way to capture and record human contact or touch and these records could give an insight as to the type of human interacting with the robot. Hopefully this will be the first step toward robots that can adapt to the individual they are interacting with, something that will greatly enhance human-robot interaction.

\section{References}

[1] The Aurora Project, http://www.auroraproject.com/, Last accessed 12/05/04.

[2] F. Michaud, and C. Théberge-Turmel "Mobile Robotic Toys and Autism", Socially Intelligent Agents - Creating Relationships with Computers and Robots, editors Dautenhahn, K. and Bond, A. and Cañamero, L. and Edmonds, B., pp. 125-132, Kluwer Academic Publishers, 2002.

[3] D. Wada, T. Shibata, T. Saito, K. Tanie "Robot Assisted Activity for Elderly People and Nurses at a Day Service Center", IEEE International Conference on Robotics and Automation, pp. 14161421, Washington, DC, 2002.

[4] B. Prazak, A. Hochgatterer, G. Kronreif, M. Furst "Robot Supported Play - New Possibilities for Physically Handicapped Children?!", AAATE 2003, Dublin, Ireland, 2003. 
[5] T. Salter, R. te Boekhorst, K. Dautenhahn, D. Jacob "Using infrared sensors for simple sensing of Robot-Human interaction styles: A preliminary study", Towards Intelligent Mobile Robots, TIMR 03. 4th British Conference on (Mobile) Robotics, UWE, Bristol, IEE, Springer Verlag London, 2003.

[6] T. Salter, R. te Boekhorst, K. Dautenhahn "Detecting and analysing children's play styles with autonomous mobile robots: A case study comparing observational data with sensor readings", IAS8, 8th Conference on Intelligent Autonomous Systems, pp. 61-70, Amsterdam, NL, IOS Press, 2004.

[7] T. Salter, K. Dautenhahn "Guidelines for robothuman environments in therapy", accepted for publication, RO-MAN 2004 13th IEEE International Workshop on Robot and Human Interactive Communication, 2004.

[8] I. Werry, K. Dautenhahn, W. Harwin "Evaluating the Response of Children with Autism to a Robot", RESNA 2001, Rehabilitation Engineering and Assistive Technology Society of North America, John Ascuaga's Nugget Hotel, Reno, Nevada, USA, 2001.

[9] B. Robins, K. Dautenhahn, R. te Boekhorst, A. Billard "Effects of repeated exposure of a humanoid robot on children with autism", Universal Access and Assistive Technology (CWUAAT), pp. 225-236, 2004.

[10] T. Fong, I. Nourbakhsh, K. Dautenhahn "A survey of socially interactive robots", Robotics and Autonomous Systems, pp. 143-166, 2003.

[11] K. Dautenhahn "The Art of Designing Socially Intelligent Agents - Science, Fiction, and the Human in the Loop", Applied Artificial Intelligence, pp. 573-617, 1998.

[12] T. Salter, K. Dautenhahn, R. te Boekhorst "Learning about Natural Robot-Human Interaction Styles", submitted manuscript, 2004.

[13] B. Jensen, G. Froidevaux, X. Greppin, A. Lorotte, L. Mayor, M. Meisser, G. Ramel, R. Siegwart "The interactive autonomous mobile system roboX", IROS 2002, IEEE/RSJ International Conference on Intelligent Robots and Systems, IEEE Press, pp. 1221-1227, 2002.

[14] 'T. Watanabe, R. Danbara, M. Okubo "Interactor: Speechdriven embodied interactive actor",
IEEE Int. Workshop on Robot and Human Interactive Communication (RO-MAN), IEEE Press, pp. $430-435,2002$.

[15] T. Kanda, T. Hirano, D. Eaton, H. Ishiguro "Interactive robots as social partners and peer tutors for children: A field trial", Journal of Human Computer Interaction (Special issues on humanrobot interaction).

[16] Wany Robotics, http://www.wanyrobotics.com/, last accessed 10-05-04, 2004.

[17] Sony, http://www.aibo-europe.com/, last accessed 10-05-04, 2004.

[18] T. Ito, NEC Personal Robot Center "How children perceive robots", http://www.incx.nec.co.jp/robot/univ/05/univ_e05.html, last accessed 10-05-04, 2003.

[19] Statsoft, http://www.statsoftinc.com/, Last accessed 12/05/04, 2004.

[20] K. Dautenhahn, I. Werry, T. Salter, R. te Boekhorst, "Towards Adaptive Autonomous Robots in Autism Therapy: Varieties of Interactions", IEEE International Symposium on Computational Intelligence in Robotics and Automation (CIRA'03), pp. 577-582, 2003. 